Das Plenum am Sonnabend-Abend griff den Dialog Jung-Alt und Ost-West wieder auf, dieses $\mathrm{Mal}$ in vollständig geglückter Form. Auf die Eingangs-Statements der vorrangig jungen Vorbereitungsgruppe aus dem Leipziger Raum hin entspann sich eine sehr lebhafte und solidarische Diskussion um Fragen nach Themenwahl und Dialogformen unter feministischen Juristinnen verschiedenen Alters und verschieden langer Einbindung in den feministischen Juristinnentag. Heiterkeit löste immer wieder das Faktum aus, dass die Grenzen zwischen jung, nicht mehr ganz so jung und älter sich durch die Diskutantinnen gar nicht so recht ziehen lassen wollten. Auch was ein West- oder ein Ost-Beitrag war, konnte, wie schon im Eröffnungsplenum, nicht ernsthaft ausgemacht werden, wenn die Redenden sich nicht selber outeten. Zumindest bei der nachwachsenden Generation scheinen sich hier bisherige Unterschiede zu verwischen. Jedenfalls die Unterschiede in der Interessenlage zwischen denjenigen, die sich schon seit Jahrzehnten mit feministischer Rechtswissenschaft auseinander setzen und denjenigen, die erst damit anfangen, zwischen Rechtsanwältinnen, Studentinnen und Lehrenden, wird teilweise im Programm bereits dadurch Rechnung getragen, dass die AGs ihre Zielgruppe ausdrücklich benennen. Und nicht nur das Rahmenprogramm, auch die gemeinsamen Diskussionen machten deutlich, was eine Studentin einmal ausdrücklich benannte: Feministische Rechtswissenschaft kann die streckenweise Monotonie und Langeweile im juristischen Studium reichlich ausgleichen, denn sie macht Lust und Spaß.

\title{
Peter Derleder/Joachim Perels Zwischen Weidenbusch und Donnersberg Zum 70. Geburtstag von Helmut Kramer
}

Ein Großteil der Mainstreamjuristen seit den soer Jahren wird ihn kaum wahrgenommen haben. Er war ein bescheidener, stiller Student der Restaurationsjahre, er war Richter und Staatsanwalt, aber nie Gerichtspräsident, Verwaltungsmann, aber ohne Chefbüro, er war Professor, aber nur vertretungsweise, er ist Autor wegweisender Arbeiten zur NS-Justiz, aber sein spektakulärstes Buch hat ein anderer geschrieben, er ist Historiker, aber mit einer Neigung zu Alltags- und Regionalgeschichte, er ist Redakteur einer Zeitschrift, die einen PR-freien Titel trägt. Und doch hat dieser keineswegs robuste Mann, der in all diesen Bereichen auch nicht mehr sein wollte, die Geschichte des bundesrepublikanischen Rechtsbewußtseins entscheidend mitgeprägt.

Geboren ist er am 30. März 1930 auf dem Kramer'schen Hof in Helmstedt. Der Vater, ein wohlhabender Landwirt, und die Klavier spielende Mutter, die ihn die Liebe zur Kammermusik lehrte, waren keine intellektuellen Leitfiguren. Aber der Vater, den er im Einspänner oft begleitete, war kein Nazi. Der Partei trat er erst bei, als er in den Vorstand der »Feldmarksinteressentschaft" gewählt werden wollte. Diese hatte für den Zustand der gemeinsamen Feldwege zu sorgen, also für die nötigen Aufschüttungen als Teil der agrarischen Produktionsbedingungen. Helmut Kramer kam mit zehn zum "Jungvolk " und wurde Ostern 1944 seiner gesetzlichen Pflicht entsprechend Mitglied der Hitlerjugend. Er war "halb dafür und halb dagegen « und marschierte bei den Jungvolkausflugsmärschen in der letzten Reihe mit. Die Lehrer 
waren Deutschnationale, die sich gelegentlich eine subkutane Distanzierung erlaub-

ten, so mit der Drohung für Unbotmäßige, daß diese eine Stunde lang den Hitlergruß entbieten müßten. Der Junge sah die amerikanischen Bombergeschwader und ihre Angriffe, erlebte, wie polnische Zwangsarbeiter kujoniert wurden, und bekam auch das Verschwinden der Säuglinge von "Fremdarbeiterinnen« mit. Er hörte den »Soldatensender Calais«, was auf dem Lande und nach der Dämmerung relativ gefahrlos war. Er verfolgte die Invasion und das Vordringen der Alliierten auf einer Karte, hörte von dem Attentat auf den Führer und glaubte zunächst einige Tage nicht an dessen Mißlingen. Der kleinste und billigste Volksempfänger (DKE 1938), die GoebbelsSchnauze, war sein medienkritisches Einstiegsobjekt. Desertierende Soldaten tauchten auf und bekamen etwas zu essen. Das Kriegsende war eine Mischung von Schrekken und Abenteuerlichem. KZ-Überlebende und Plünderer prägten sich ins $\mathrm{Ge}$ dächtnis ein. An der nahen SBZ-Grenze kam es zu Verbrechen an Grenzgängern, die von ortskundigen »Fluchthelfern« ausgeraubt und manchmal vergewaltigt und ermordet wurden. Helmut Kramer spürte auch die vorübergehende Aufbruchstimmung nach dem Krieg. Seine Lehrer bis zum Abitur 1949 waren überwiegend mäßig. Einer unterhielt zeitweise eine Korrespondenz mit dem Schriftsteller Georg Britting, aus der er gerne vorlas.

Nach dem Abitur im Jahre 1949 begann Helmut Kramer sofort mit einem Studium generale in Wilhelmshaven-Rüstersiel, wo Wolfgang Abendroth und Rüdiger Altmann gleichzeitig anzutreffen waren. Nach einem Semester ging er für ein Jahr nach Götringen und studierte außer Geschichte auch Kunstgeschichte. Seminare zu Bildern von Claude Lorrain berührten die Geheimnisse der Landschaftsästhetik. Dann (I95I) kam der Wechsel nach Freiburg in das Brotberufsstudium Jura, den der Vater gerne sah. Zu lesen war jetzt der schreckliche "Allgemeine Teil " von Heinrich Lehmann, der Alptraum eines didaktikfreien Rechtsstudiums, und immer wieder das Strafrecht von Welzel, der mit den Ontologismen der Finalen Handlungslehre den Rückblick auf das NS-Strafrecht verdrängte. Freiburg bot Erik Wolf, der Mitmacher gewesen, aber zur Bekennenden Kirche gekommen war, Gustav Boehmer, Werner Thieme, Wilhelm Grewe und Ernst Rudolf Huber, der, Schüler und Mitarbeiter Carl Schmitts, die "Verfassung des Großdeutschen Reiches « geschrieben hatte. Die Nazirechtsliteratur war sorgfältig durch Verwahrung in »Giftschränken« unzugänglich gemacht. Helmut Kramer hat sich das alles erst später klargemacht. Die Nazivergangenheit der Hochschullehrer blieb im Bereich des On-dit. "Heidegger liest wieder «, hieß es, er konnte der Vorlesung keine Botschaft entnehmen. Im Examen fragte ihn Hans Thieme nach den Schöppenstühlen, und der Prüfling befriedigte das professorale Selbstverständnis durch ein Lob auf die Bedeutung des Rechtsgutachtens.

Als Doktorvater suchte sich Helmut Kramer nach dem Referendarexamen i 955 der Präzision und des Anspruchs wegen Ernst Rudolf Huber aus. Aus der Verfassungsgeschichte der Neuzeit erhielt er nach dem ersten Stadium bundesrepublikanischer Parteiendemokratie als Thema den Fraktionszwang. Wie man mit Quellen umging, mußte er sich selbst beibringen. Kopien hatten die Lesequalität der N'quam-Rollen und kosteten so viel wie heute ein Toast Hawaii. Er begann mit Weimar, seinen Zeitungen und Fachzeitschriften, und stieß ins 19. Jahrhundert vor. Die Arbeit nahm über Referendar- und Gerichtsassessorzeit einschließlich längerer wissenschaftlicher Urlaubsphasen über ein Jahrzehnt lang in Anspruch. Er studierte im Frankfurter Bundesarchiv die Paulskirchendokumente, die Genesis der deutschen parlamentarischen Demokratie, mit ihren Clubs genannten Parteien. Im Weidenbuschclub traf sich die Mittelgruppierung einschließlich des Westendhallclubs ("Linke im Frack «), während der Donnersbergclub die äußerste Linke darstellte, die für Volkssouveräni- 
tät und Republik eintrat. Es gab durchaus Beispiele massiven Fraktionszwangs, wie auch schon im Vormärz, im badischen Landtag. Der Abgeordnete Eisenmann glaubte, dem Dilemma zwischen Gewissensfreiheit und Gruppenzwang mit der Gründung eines Ein-Mann-Clubs entgehen zu können. Helmut Kramer stöberte die rigiden Satzungen einiger Clubs auf. Der Doktorand hatte sich ergänzend durch die reiche Memoirenliteratur zu lesen. Der Konflikt zwischen Überzeugungstreue und Anpassung begleitete ihn durch die gesamte Arbeit, während seiner Freiburger Referendarzeit, nach der Rückkehr ins Niedersächsische, über das Assessorexamen in Hannover 196I hinaus. Danach wollte er zunächst mit elterlicher Alimentation die Dissertation abschließen, obwohl die Justiz aufgrund seines guten Examens Interesse an ihm zeigte. 1962 wurde er schließlich Gerichtsassessor, heiratete und lieferte im Geburtsjahr des ersten der beiden Kinder 1965 das Opus schließlich ab. Es lag einige Zeit bei Huber, der ihm dann aber plötzlich das Gelingen telefonisch attestieren ließ. Heute gehört die Schrift zu den rechtsgeschichtlichen Klassikern aus den Anfängen der Parteiendemokratie und würde eine Neuauflage verdienen (»Fraktionsbindungen in den deutschen Volksvertretungen 1819-1849", Duncker \& Humblot, I968). Das gesamte, zum Teil unersetzliche Forschungsmaterial seit 1849 warf der Bescheidene weg, um das Trauma der Bearbeitungsdauer loszuwerden.

Als Gerichtsassessor in der Strafjustiz hatte es Helmut Kramer bald mit entschlossener NS-Amnesie und den Strafexerzitien des Kalten Krieges zu tun. Bei der Generalstaatsanwaltschaft, wo er als vielversprechender Nachwuchsjurist eingesetzt wurde, betraf 1965 eine der ersten Akten ein Todesurteil aus dem Jahre 1944, das gegen Erna Wazinski. Diese 19jährige hatte, selbst ausgebombt, aus Gebäudetrümmern einen fremden Koffer mit wenig wertvollem Inhalt mitgenommen. Ihre Mutter hatte nach 1945 mit der Obstinatheit eines nicht auszulöschenden Schmerzes versucht, ihr dafür von einem Sondergericht als »Volksschädling " zu Tode verurteiltes und in Wolfenbüttel hingerichtetes Kind rehabilitieren zu lassen. In Braunschweigs Justiz, in der wie andernorts nicht wenige ehemalige Nazirichter mit ihrem unbehelligten besten Gewissen Recht pflegten, gab es für diese Mutter kein Erbarmen. Eine Strafkammer erklärte 1965 nach langem Zuständigkeitsstreit sogar in einem kaum mehr überbietbaren Begründungsexzeß das Sondergerichtsurteil nachträglich für zwingend geboten. Die Volksschädlingsverordnung, das Kernstück des NS-Unrechtsstaates für den deutschen Kriegsalltag, provozierte noch einmal eine Art virtueller Täterjustiz. Gerichtsassessor Kramer, der auf diese Justiz nicht vorbereitet war, votierte vergeblich für eine Entschädigung der Mutter. Der Vorsitzende des für das Todesurteil verantwortlichen Sondergerichts, Werner Lerche, wurde nach dem Krieg hoher kirchlicher Amtsträger und erhielt bei seinem Tod 1962 von der braunschweigischen Landeskirche einen ganzseitigen Nachruf auf sein "gesegnetes" Leben. Der Berichterstatter für das 196 ser Urteil wurde später Bundesrichter.

Besonders gut funktionierten alte NS-Juristen verständlicherweise im politischen Strafrecht mit seinen diffusen Tatbeständen und seinen bombastischen Strafdrohungen. Die Straftat der »landesverräterischen Beziehungen« nach $\$$ rooe StGB erfaßte schon harmlose Mitteilungen an einen »von drüben" wie etwa diejenige, daß die Kasernen in Braunschweig vier Stockwerke hatten. Helmut Kramer hatte die Anklageschrift gegen einen wenig intelligenten Bundeswehrangehörigen mit einem ähnlichen Ostkontakt zu schreiben und plädierte für eine sechsmonatige Bewährungsstrafe. Das Urteil lautete dann auf ein Jahr ohne Bewährung, nicht ohne bedeutungsvolle Geste des Senatspräsidenten Meier-Branecke für den jungen Kollegen. Meier-Branecke, vom Stahlhelm 1933 zur NSDAP übergelaufen, am Ende des Krieges "Leiter der gnadenlosen Gnadenabteilung im Oberkommando des Heeres" (Ingo Müller), was nicht ohne den Nachweis rückhaltlosen Einsatzes für den natio- 
nalsozialistischen Staat möglich gewesen war, war nach dem Krieg in Braunschweig schnell zum Senatspräsidenten aufgestiegen, konnte bruchlos weiter seiner Disposition für hohe Strafen und gegen Verweichlichung frönen. Helmut Kramer waren diese Zusammenhänge zunächst ebensowenig geläufig wie seinen Altersgenossen in der Kollegenschaft. Die Schlüsselerlebnisse von damals aber wirkten lange nach. Er begann, die Karrieren der NS-Richter zu rekonstruieren, in einem Stadium der Nachkriegsgeschichte, als dies noch als Nestbeschmutzung galt. Nur der Frankfurter Generalstaatsanwalt Fritz Bauer versuchte nachdrücklich, Licht in das mörderische Dunkel des NS-Justiz zu bringen. Bei der Generalstaatsanwaltschaft hatte Helmut Kramer 1965 Berührung mit den von Bauer erstellten Akten für ein Verfahren gegen die Spitzen der NS-Justiz wegen der Unterstützung des Anstaltsmords.

Mitte 1968 wechselte Helmut Kramer zur Ziviljustiz, verbrachte eine Probezeit beim OLG Braunschweig und wurde 1972 Landgerichtsdirektor mit einer kleinen Strafkammer. Als Rudolf Wassermann als OLG-Präsident in Braunschweig vorgeschlagen wurde, schrieb Helmut Kramer einen Leserbrief gegen dessen Perhorreszierung. Die Begegnung mit der 68er Bewegung und dem Reformer Wassermann ermutigte ihn zur Fortsetzung seiner publizistischen Tätigkeit. Er schrieb einen Artikel im Handlexikon von Axel Görlitz, diesem Brevier junger kritischer Juristen, zum Gerichtsverfassungsrecht. Er trat aus dem damals ständisch-konservativen Richterbund aus, gründete 1972 mit drei Kollegen die niedersächsische Fachgruppe »Richter und Staatsanwälte in der ÖTV « und gibt seither die Zeitschrift »ÖTV in der Rechtspflege« mit heraus. Das Verständnis von Rechtspflege als öffentlicher Dienstleistung galt damals als Provokation. Er engagierte sich in Wassermanns Fahrwasser bei der Justiz wie im niedersächsischen Justizministerium für eine Pluralisierung der Richterschaft und für das neue Fach Rechtskunde. In der allzu früh eingestellten, weiterhin aber äußerst lesenswerten Zeitschrift »Recht und Gesellschaft« wurden die Problemfelder der Rechtsordnung in vorbildlicher Weise für den Unterricht mit Schülern aufbereitet. Die Arbeit der 7oer Jahre brachte Helmut Kramer vielfältigen freundschaftlichen Konnex auf der Basis der reformerischen Ideen. Bei einer Fortbildungstagung über »Die juristische Sprache der Gerichte als Machtmittel « lernte er die Referendarin Barbara Kriege kennen, weiblichen »Nachwuchs in der ÖTV«, die sich mit ihm dagegen stemmte, daß die Tagungsteilnehmer vorzeitig verschwanden, um die tschechische Eishockeymannschaft siegen zu sehen. Es dauerte dann aber noch geraume Zeit, bis es zu einer Verbindung, gar einer ehelichen, kam, aus der ein Sohn hervorgegangen ist.

Mit Wassermann, für den Helmut Kramer die Verwaltungsarbeit des OLG erledigte, kam es in der Puvogel-Affäre 1978 schließlich zum Bruch. Im Kabinett des niedersächsischen Ministerpräsidenten Albrecht saß Provinzanwalt Puvogel als Justizminister, der in seiner Dissertation von 1937 die Minderwertigkeit der »für die Gemeinschaft nutzlosen", rassisch nicht wertvollen Menschen behandelt und deren »Ausmerzung " gefordert hatte. Albrecht, der in einer frühen Schrift die Folter im Staatsnotstand legitimiert, dies aber auf offentlichen Druck zurückgezogen hatte, war nicht erkennbar schockiert. Puvogel selbst verteidigte sich zunächst auf die ihm eigene Art, ohne Reflexion und Intellekt. Helmut Kramer verschickte Textauszüge aus der Dissertation ohne Absenderangabe unkommentiert an prominente Justizkollegen. Ein Kommentar war auch nicht nötig. Puvogel wurde wieder Anwalt. Auf Albrechts Weisung hatte nun aber OLG-Präsident Wassermann den Täter, also den Absender, zu ermitteln und disziplinarisch zu ahnden. Wassermann war nicht eingeweiht, wußte aber sofort, das konnte nur Kramer sein. Das Disziplinarverfahren führte zu keiner Strafe, doch wurde eine Dienstpflichtverletzung festgestellt. Dem Einstellungsbescheid war die gewandte Feder Wassermanns nicht anzumerken. »Es 
steht dem Richter ebensowenig wie dem Beamten zu, seinem Vorgesetzten Verfehlungen vorzuwerfen, « hieß es unter Berufung auf einen Kommentar aus der Adenauerzeit. Eine Wahrnehmung berechtigter Interessen wurde Kramer mangels »krisenhaft zugespitzter Situation « nicht zugebilligt. Helmut Kramer nahm den Bescheid hin, weil er die fehlende Absenderangabe bedauerte. Wassermann, einer der bedeutendsten Justizreformer der Bundesrepublik, ein kritischer Rechtssoziologe der ersten Stunde, dem die Konservativen in der Justiz lange Jahre in bitterer Weise die Anerkennung versagt hatten, stand Helmut Kramer seitdem ferner und wurde in der Folgezeit allmählich immer konservativer. Freilich überrascht auch der "Welt«- und NJW-Autor gelegentlich noch durch den Rückgriff auf Erkenntnisse aus seiner politischen Wirkungszeit.

Im Jahr der Puvogel-Affäre, 1978, schreckte Helmut Kramer eines Nachts auf mit dem Bild einer NS-Akte, die er zuletzt 1965 in Händen gehabt hatte, beim Kontakt mit Fritz Bauers Recherchen. Es war mühsam, das Aktenzeichen zu ermitteln, und noch mühsamer, an die Akten heranzukommen, deren Herausgabe der Frankfurter Generalstaatsanwalt jahrelang verweigerte. Es gab auch vor der Entdeckung des Grundrechts auf informationelle Selbstbestimmung umfassenden Datenschutz, hauptsächlich für NS-Belastete. Schließlich wurden Helmut Kramer 98 Seiten von I4 Bänden mit mehr als 3000 Seiten aus dem Archiv überlassen. Daraus entstand der Bericht über die Einbindung der deutschen Justiz in Hitlers Programm zur heimtükkischen Tötung der Geisteskranken (KJ 1984, 25 ff.).

Das Führungskorps der Justiz, vom Reichsgerichtspräsidenten Bumke bis zu den 34 OLG-Präsidenten, hatte am 23./24. April $194 \mathrm{I}$ im Haus der Flieger in Berlin schweigend die Anweisungen zur geheimgehaltenen "Aktion $\mathrm{T}_{4}$ « entgegengenommen und sich in die reibungslose Organisation einspannen lassen. Nur ein Teilnehmer bat um Erlaß eines Gesetzes. Im Nürnberger Ärzteprozeß waren die beiden Hauptorganisatoren zum Tode verurteilt worden. Der psychiatrische Protagonist, Prof. Dr. Werner Heyde, war als Dr. Sawade 12 Jahre lang insbesondere von der schleswig-holsteinischen Sozialgerichtsbarkeit gedeckt worden. Fritz Bauer betrieb in den $60 e r$ Jahren die Verfolgung von I I OLG-Präsidenten und s Generalstaatsanwälten wegen Beihilfe zur Massentötung Geisteskranker. Die Justiz aber verschleppte das Verfahren, die Angeschuldigten gaben sich moribund. Nach Fritz Bauers Tod 1968 war plötzlich die Chance da, einen "Schlußstrich« zu ziehen. Bauers Nachfolger Gauf zeigte kein erkennbares Interesse, auch wenn er sich nachträglich in wenig überzeugender Weise rechtfertigte. Auf 9 Zeilen setzte das LG Limburg 1970 dann die Angeschuldigten außer Verfolgung. Diese hatten sich unter anderem damit verteidigt, für sie sei die Aktion »von bestürzender Neuheit « gewesen, ihnen härte eine Schreckund Reaktionszeit zugebilligt werden müssen. Der angeschuldigte Generalstaatsanwalt Dr. Friedrich Walter Jung behauptete, er habe später dem Staatssekretär Schlegelberger mündlich seine religiösen Bedenken vorgetragen. Einen Vermerk darüber gab es nicht. Eine Strafanzeige wie Graf von Galen, Bischof von Münster, erstattete keiner gegen die Anstaltsmörder. Statt dessen leiteten sie die eingehenden Strafanzeigen aus der Bevölkerung sicher weisungsgemäß in den NS-Papierkorb. Erst Helmut Kramers Forschungsarbeit erhielt die Straflosstellung dieser Hüter des Unrechts dem kollektiven Gedächtnis.

Die Publikation hatte noch ein Nachspiel. Der Sohn des angeschuldigten Generalstaatsanwalts Jung, selbst Botschafter in Budapest, beschuldigte Kramer der unwürdigen Tatsachenfälschung. Der Bonner Staatsanwalt Hundertmark leitete deswegen ein Strafverfahren gegen Botschafter Jung ein, in dem man die historische Wahrheit gerichtlich hätte feststellen können. Die dafür wesentlichen Is Bände des Frankfurter Ermittlungsverfahrens verschwanden jedoch plötzlich. Die Justiz beschränkte sich 
lange Zeit auf die Suche nach ihnen. Als diese in einem Postpaket ohne Absenderangabe endlich wieder auftauchten, wurde Jung vom Amtsgericht Bonn freigesprochen, nicht zuletzt wegen unvermeidbaren Verbotsirrtums. In der Berufungsinstanz kam es zunächst zu einer rechtswidrigen, 1990 dann zu einer endgültigen Einstellung, wobei das Gericht jede historische Aufklärung zwanghaft zu vermeiden suchte. Heinrich Hannover, der Helmut Kramer prozessualen Beistand leistete, hat in seinen Erinnerungen "Die Republik vor Gericht" (1975-1995, S. 307) darüber berichtet. Statt der Erörterung der historischen Wahrheit gelang der Justiz nur wieder eine Posse.

Dem eigenen Werdegang und der Berührung mit den NS-Justizprotagonisten geschuldet war die regionale Justizgeschichte. "Braunschweig unterm Hakenkreuz " ( $198 \mathrm{I}$ ) - für die Stadt, in der Hitler Regierungsrat und damit deutscher Staatsbürger geworden war - war ein aus einer Vortragsreihe hervorgegangener Band, mit dem Helmut Kramer dem Tucholsky-Motto folgte: »Wie mache ich mich unbeliebt? «. Er rollte die örtlichen Justizkarrieren auf, die von den NS-Todesurteilen direkt in die Nachkriegsrechtspflege geführt hatten. Wer wie der NS-OLG-Präsident Nebelung sogar zum Volksgerichtshof gelangt war (dabei konnte man wie ein freundlicher Daddy aussehen), durfte zwar nicht weiter amtieren, aber als Anwalt und Notar mit Richterpension im juristischen Erwerbsleben untertauchen. In diesem von Helmut Kramer herausgegebenen Buch wurde auch rekonstruiert, wie die Nachkriegsjustiz die Aufarbeitung der Vergangenheit hintertrieben hatte.

Die Täter zu verstecken, hieß auch die Spuren der Opfer zu tilgen. In Wolfenbüttel sollte demgemäß die ehemalige Hinrichtungsstätte auf dem Gelände des Gefängnisses abgerissen werden, eine im Jahr 1937 als Beitrag des Reichsjustizministeriums zum geplanten Angriffskrieg errichtetes Gebäude, mit Uhren- und Glockenturm, von dem - mitten in dem behaglichen Bürgerstädtchen - der Rhythmus des Mordens ausging. Hier waren (meist nach qualvoller Gefangenschaft) Hunderte hingerichtet worden, Verfolgte des Naziregimes und viele Widerstandskämpfer aus den Ländern Westeuropas. Hier war das Zentrum der Henkerarbeit in Norddeutschland, dessen Bausubstanz unversehrt, dessen Innenraum aber verkommen war, obwohl Hinterbliebene hier oft ihrer Toten gedachten. Der Justizminister bot eine Gedenktafel als Ersatz an, da sich ein Abriß nicht vermeiden lasse. Helmut Kramer mobilisierte die Veteranenverbände, insbesondere auch die französischen und belgischen mit ihrer jeweils unterschiedlichen politischen Couleur. Diesen mußte nicht nur auf Französisch der Sachverhalt verdeutlicht, sondern auch wirkungsvolle Formulierungshilfe geboten werden, bis schließlich Bundespräsident von Weizsäcker dem Präsidenten der Internationalen Föderation der Widerstandskämpfer, Arialdo Banfi, melden konnte, es habe nur »unverbindliche Überlegungen« zum Abriß gegeben, jetzt aber sei gesichert, daß die ehemalige Hinrichtungsstätte in einen würdigen Zustand versetzt werde. Justizminister Remmers bestätigte dann auch nachträglich, schon früh sei erkannt worden, daß der Platz doch nicht zur Sanierung der Wäscherei genutzt werden, sondern zur Gedenkstätte gemacht werden müsse. Diese wurde dann 1990 eröffnet und mit einer Dauerausstellung über die Leiden der mit Guillotine und Strang Hingerichteten verbunden. Besonders eindrucksvoll war das Werk der Uhr, das den zum Tod Verurteilten die Stunde schlug. Hier war auch Erna Wazinski unterm Fallbeil gestorben. Ihren Abschiedsbrief fand ein Zeitzeuge: „Geliebte Mutti, wenn $\mathrm{Du}$ diesen Brief erhältst, bin ich tot. Sei tapfer! Wir werden uns ja einmal wiedersehen. Verzeiht mir und behaltet mich lieb ... «

199I erwirkten Helmut und Barbara Kramer schließlich in einem Wiederaufnahmeverfahren den Freispruch für Erna Wazinski. Der Vorsitzende Richter lehnte die Bestellung von Barbara Kramer zur Pflichtverteidigerin allerdings mit der Begrün- 
dung ab, daß sie sich zu lange mit der Sache beschäftigt habe. Ohne jahrelanges Bemühen wäre dieser Richter mit der Sache gar nicht befaßt worden. Ohne Helmut Kramers grundlegende Forschungsarbeiten und seine selbstlose Bereitwilligkeit, eine Fülle eigener Materialien zur Verfügung zu stellen, wäre auch Ingo Müllers Buch "Furchtbare Juristen « (1987) nicht möglich gewesen, ein Buch, dessen hohe schriftstellerische Qualität sich vor allem der lakonischen kommentarlosen Präsentation der Fakten und Materialien verdankt.

In dem Jahrfünft (1984-1989), in dem Helmut Kramer Vertretungsprofessor am rechtswissenschaftlichen Fachbereich der Universität Bremen war, fand seine Arbeit endlich eine vorbehaltlose Resonanz, also ohne das verdeckte Ressentiment, das ihm meist in der Justiz begegnet war. Seine rechtshistorischen Vorlesungen waren eine besondere Attraktion, aufgrund der (auch mit Medieneinsatz) unternommenen Rekonstruktion der NS-Justizverbrechen. Daneben widmete er sich in Anknüpfung an seine vorausgegangene richterliche Tätigkeit speziell dem Familienrecht, wo er dem Vertiefungsstudium Impulse gab. Mit der Bahn aus Wolfenbüttel gekommen schwang er sich samt seinem stets reichhaltigen Materialienpaket auf ein permanent am Hauptbahnhof geparktes rostiges Fahrrad, das ihn nicht immer wohlbehalten zu den Hörsälen jenseits des Stadtwaldes brachte und das gelegentlich doch infolge des Zugriffs eines allzu bescheidenen Diebes ersetzt werden mußte. Als Lehrer war er durch eine spezielle Synthese von äußerster Detailakribie und übergreifender Gedankenentwicklung geprägt, zwischen denen die Assoziationen hin und her flogen. Die damit notwendig verbundene professorale Zerstreutheit provozierte auf Seiten der Studierenden eine geradezu liebevolle Aufmerksamkeit, die für den Fall des Verlusts eines Materialienpakets eigene Programme für seine Erörterung in petto hatten.

In die Bremer Zeit fielen die politischen Auseinandersetzungen um die Realisierung der noch von Helmut Schmidt initiierten Nachrüstung durch Stationierung von Atomwaffen bei Mutlangen und anderswo, die der Eigenlogik eines führbaren Atomkrieges und der Kalkulation mit dem Overkill folgte. Die gewerkschaftlich organisierten Richter und weitere Mitglieder des auch von Helmut Kramer inspirierten »Richterratschlags « beteiligten sich an den Protesten der Friedensbewegung. Helmut Kramer »nötigte« am I2. Januar 1987 bei ca. 20 Grad Minus mit einigen anderen Demonstranten einen US-Militärlaster bei Mutlangen zu kurzem Halt und wurde trotz der öffentlichen Solidaritätsadresse von mehr als soo Kolleginnen und Kollegen ("Beendet den Wahnsinn der atomaren Rüstung «) vor dem Amtsgericht SchwäbischGmünd angeklagt. Während sich der Großteil der angeklagten Richter und Staatsanwälte mit gesinnungsethischer Wortkargheit verurteilen lassen wollte, entfaltete Helmut Kramer einen hochdifferenzierten rechtshistorischen, rechtsdogmatischen und rechtspolitischen Verteidigungsdiskurs, der nicht nur zum Rückzug des ersten zuständigen Richters, sondern auch dazu führte, daß ihm sein Strafverteidiger, der damalige Oppositionsführer im niedersächsischen Landtag, Gerhard Schröder, immer wieder besänftigend von hinten die Hand auf die Schulter legte und selbst kaum zu Wort kam. Konterpart war der in diesen Prozessen zum Provinzphilosophen gereifte Richter Offenloch, der sich nicht scheute, notfalls unter Berufung auf Kant der atomaren Vernichtung das noch größere Übel der Anarchie mit Sitzblockaden abwägend entgegenzuhalten. Die spätere Änderung der Rechtsprechung durch BVerfG zu $\$ 24^{\circ} \mathrm{StGB}$ muß ein tantalischer Schmerz für diesen gewesen sein. Offenloch erkannte jedenfalls gegen Helmut Kramer auf 20 Tagessätze à 100 DM. In der Rechtsmittelinstanz kam es aufgrund einer Entscheidung des BVerfG dann zur Verfahrenseinstellung. 
Nach der Rückkehr von der Bremer Professur erhielt er von der rot-grünen Koalition in Niedersachsen den Auftrag zur Aufarbeitung der jüngeren Justizgeschichte des Landes. Gegen manche Widerstände realisierte er die regelmäßige Tagung der Deutschen Richterakademie zu diesem Thema. 1994 erhielt er in Hamburg den HansLitten-Preis der VDJ, der an einen der mutigsten Rechtsanwälte der Weimarer Republik erinnert. Auch nach dem Eintritt in den Ruhestand bekämpfte Helmut Kramer unerbittlich die wieder aufkommende Geschichtsvergessenheit, so etwa, wenn eine Landeszentrale für politische Bildung ihre wissenschaftliche Neugier bei der Neuen Rechten befriedigte. Seine rechtsgeschichtliche Arbeit mündete 1999 in die Gründung eines Forums Justizgeschichte (vgl. KJ 1999, 333ff.), das eine feste Tradition begründen soll, bevor Martin Walsers Themamüdigkeit und Peters Sloterdijks gelockerte Denkart um sich greifen.

Aus der Beschäftigung mit der Geschichte leitet Helmut Kramer auch die Verpflichtung zur kritischen Begleitung des gegenwärtigen Zeitgeschehens ab. Die völkerrechtliche Grundsatzkritik an der neuen Nato-Doktrin hat ihn bewogen, in Berliner Strafprozessen die Verteidigung von Pazifisten zu übernehmen, die den Kriegsdienst im Konflikt mit Jugoslawien verweigert haben.

Helmut Kramer hat in Anknüpfung an Fritz Bauer, zusammen vor allem mit Manfred Messerschmidt, Michael Stolleis und Ingo Müller, einen entscheidenden Beitrag zur kollektiven Erinnerung an die äußerste Perversion des Rechts geleistet. Er war der älteste, er ist als Einzelner angetreten wie auch der Abgeordnete Eisenmann während der $48 \mathrm{er}$ Revolution und hat davon zumindest bei seinen Aktionen immer etwas bewahrt, auch wenn er seit 1972 viel Solidarität gefunden hat. Er hat sich nie mit der Rolle des reinen Idealisten begnügt, der aus besserer Zeit zurückblickt, sondern die Klitterer und Leugner treffsicher traktiert. Er, der Kritiker der Justiz, hat vor ihrer Erledigungsmentalität die sprechendsten Fakten gerettet und sich dabei paradoxerweise auch immer wieder in (letztlich oft nicht ganz unergiebige) gerichtliche Kleinkriege verstrickt wie zuletzt denjenigen darüber, ob er (auch) durch das Wiederaufnahmeverfahren für Erna Wazinski gegen das aus der NS-Zeit stammende Rechtsberatungsgesetz verstoßen hat. Aufgrund einer entsprechenden Selbstanzeige wurde er verurteilt. Mit einem solchen Selbstversuch als Entlarvungsmodus will er darauf aufmerksam machen, daß eine historisch ignorante Justiz dieses Gesetz sogar als Verbot unentgeltlicher Rechtsberatung von Erniedrigten und Beleidigten versteht. Der Gedanke, daß er im Gegensatz zu ihm Nahestehenden der Barbarei (wenn auch knapp) entronnen ist und daß die Abwehr dieser Geschichte das demokratische und soziale Gerüst der Gesellschaft nach wie vor in Frage stellt, verursacht bei ihm bis heute die Unruhe des Herzens, die darauf hoffen läßt, daß er auch das unter seinen Händen entstandene einmalige Archiv juristischer Zeitgeschichte in seinem Hause noch in Publikationen auswertet. 